\title{
Are new genome variants detected in SARS-CoV-2 expected considering population dynamics in viruses?
}

\author{
Mahmut Cerkez Ergoren ${ }^{1,2^{*}}$, Pinar Tulay ${ }^{1,2}$, Munis Dundar $^{3}$
}

\begin{abstract}
The pandemic COVID-19 is caused by a highly transmissible severe acute respiratory coronavirus 2 (SARS-CoV-2) which showed the highest morbidity and mortality rates among the other coronavirus infections such as SARS-CoV and MERS-CoV. However, the numbers of infected cases as well as mortality rates are varying from population to population. Therefore, scientist has urged the SARS-CoV-2 genome and host genetic factors investigations. Recently, new SARS-CoV-2 variants has been detected and though to affect the diseases transmission from human to human. In this mini-review, we aimed to explained detected SARS-CoV-2 variants that thought to influence the COVID-19 severity and transmission using the literature.
\end{abstract}

Keywords: SARS-CoV-2; variant; ACE2; coronavirus

${ }^{1}$ Near East University, Faculty of Medicine,

Department of Medical Genetics, Nicosia North,

Cyprus

${ }^{2}$ Near East University, DESAM Institute, Nicosia North, Cyprus

${ }^{3}$ Erciyes University, Faculty of Medicine, Department of Medical Genetics, Kayseri, Turkey

\section{${ }^{*}$ Corresponding author:}

Assoc. Prof. Mahmut Cerkez Ergoren, Ph.D. Near East University, Faculty of Medicine,

Department of Medical Genetics

Near East University, DESAM Institute, Nicosia,

Cyprus

Near East Boulvard, Nicosia North, 99138, Cyprus

E-mail address: mahmutcerkez.ergoren@neu.edu.tr

DOI: 10.2478/ebtj-2021-0001

(C) 2021 Authors. This work was licensed under the Creative Commons Attribution-NonCommercial-

NoDerivs 4.0 License.

\section{Introduction}

The $21^{\text {st }}$ century has been faced twice to Sarbecoviruses causing epidemics and pandemics globally (1). The strain of $\beta$-coronavirus, severe acute respiratory syndrome coronavirus 2 (SARS-CoV-2), causes the ongoing pandemic coronavirus diseases 2019 (COVID-19) which was first reported in Wuhan, China in December 2019. Since then, SARS-CoV-2 has spread to the planet rapidly. The World Health Organization (WHO) declared that this current COVID-19 outbreak infected approximately 88 million confirmed cases with more than 1.9 million deaths worldwide by January 9, 2021. Previosuly, mutations within the viral genome has shown to alter the RNA virus transmissibility, virulance and pathogenesis (2). Due to the natural proces recombination coronaviruses (SARS-CoV and SARS-CoV-2) may undergo frequent recombination resulting structural genetic diversity. However, SARS-CoV-2 is not a recombinat of any sarbecoviruses to date. Its specific binding ability to human Angiotensin-Converting Enzyme 2 (ACE2) receptor shows to be shared ancestral trait with two severe acute respiratory syndrome (SARS)-like bat coronaviruses, bat-SL-CoVZC45 and bat-CoVZXC2, but there is no direct evidence and molecular mechanisms still need to be resolved $(3,4)$.

The SARS-CoV-2 genome consists of 30 kilo base $(\mathrm{kb})$ including both coding and non-coding structural protein regions. Envelope (E), spike (S), and nucleocapsid $(\mathrm{N})$ and membrane (M) are SARS-CoV-2 structural proteins (5). S protein is located on the surface of the virus and interact with human ACE2 receptors as well as has a key role in human to human transmission (6). The fatality rate of the SARS-CoV-2 infection ( $44 \%)$ still is higher than SARS-CoV (9.6\%) and MERS-CoV (34.3\%) (7). Therefore, the effective treatment is urged to decrease the COVID-19 mortality and morbidity rates. The mortality rates of the COVID-19 infection is shown to differ region to region. Therefore, SARS$\mathrm{CoV}-2$ genetic variations and human genetic markers might affect the severity rate of the COVID-19, up to now have not been clearly understood. In this mini-review, we focused on the SARS-CoV-2 variants and their possible effects on the host using the information 
from the literature.

\section{SARS-CoV-2 genome variants}

In spite of, the CoV RNA polymerase proofreading function higher replication accuracy was indicated by genetic epidemiologic studies. In late February SARS-CoV-2-associated D614G variant was detected in South Europe, since then has rapidly spread worldwide and replaced the original SARS-CoV-2 strain. A higher viral load as well as increased transmission activity were observed in COVID-19 cases with the D614G variant than the cases without the D614G mutation (8). However, SARS-CoV-2-associated D614G substitution does not influence the severity and affect the treatment methodology of the diseases.

The SARS-COv-2 variant named as "Cluster 5 " was detected in farmed minks associated with the infection and afterwards transmitted to humans by Danish authorities. Fortunately, only 12 cases have been confirmed by this variant to dates, clarifying that the variant does not spread globally (9). On December, $14^{\text {th }}$ 2021 British authorities announced a new SARS-CoV-2 variant called as SARS-CoV-2 VOC 202012/01 to WHO. Interestingly, this variant has a combination of 23 nucleotide substitutions, phylogenetically differ from the SARS-CoV-2 that circulate in the United Kingdom and showed increase transmission activity (10). Since then, more than 31 countries have reported the new SARS-CoV-2 variant to the Global Initiative on Sharing Avian Influenza Data (GISIAD) initiative. However, studies indicated that there is no diseases severity in the illness (11). On the other hand, a mutation named as the 69/70del within the $S$ gene of SARS-CoV-2 VOC 202012/21 variant was found to alter the real-time polymerase chain reaction (RT-PCR) performance related in the diagnostic purposes but it does not change the performance of antigen-based detection. Late December 2020, phylogenetically different virus variant 501.YV2 was detected and rapidly speeded in South Africa. It worth to mention that the VOC202012/21 variant also has the N501Y mutation. However, there is no certain evidence of the new 501.YV2 variant affect the diseases severity or other frightening outcomes.

\section{Discussion}

Despite SARS-CoV-2 has a much higher transmission and spread rate than known other coronavirus strains, the reports indicated that the most of the cases do not show any symptoms (asymptomatic). COVID-19 might easily transmitted from human to human if the environment is suitable. When the virus enters this nasal path, it begins to spread to the sinuses in the nasal cavity, nasal mucosa, pharynx area, trachea and lungs. It should not be forgotten that the virus must enter the cell in order to survive. It has been shown that $\mathrm{S}$ protein of the CoVs bind to human ACE2 receptor to enter the host via endocytosis. The study comparative genetic analysis study conducted by Cao et al. (2020) was showed no direct evidence to support the presence of ACE2 variant resistance to coronavirus S-protein binding among World populations. However, in the same study there were structures associated with the presence of higher
ACE2 protein expression in tissues in East Asian populations (12). However, the genome-wide association studies that aimed to show the putative human genetic susceptibility factors to COVID-19 infection have been conducted, but there is no direct evidence up-to-date (13-18).

Overall, the current SARS-CoV-2 variants might be differing according to transmission ability as still is SARS-Cov-2, and it does not seem to affect the severity of the illness. We believe it will not alter to current treatment strategies as well as vaccine developments. However, human genetic variants especially on B and T cell receptors as well as ACE2 and TMPRSS2 proteins should be considered while designing the therapeutics to decrease the transmission of the diseases. Therefore, precision medicine time has come to help humans to survive this war with the novel coronavirus.

\section{References}

1. Baric RS. Emergence of a Highly Fit SARS-CoV-2 Variant. New England Journal of Medicine. 2020: 383(27); 26842686.

2. Lam TT-Y, Zhu H, Guan Y, Holmes EC. Genomic analysis of the emergence, evolution, and spread of human respiratory RNA viruses. Annu Rev Genomics Hum Genet 2016;17: 193-218.

3. Boni MF, Lemey P, Jiang X, et al. Evolutionary origins of the SARS-CoV-2 sarbecovirus lineage responsible for the COVID-19 pandemic. Nat Microbiol. 2020; 5: 1408-1417

4. Liu K, Tan S, Niu S, et al. Cross-species recognition of SARS-CoV-2 to bat ACE2. Proceedings of the National Academy of Sciences. 2021: 118(1), e2020216118.

5. Wu F, Zhao S, Yu B, Chen YM, Wang W, Song ZG, et al. A new coronavirus associated with human respiratory disease in China. Nature. 2020; 579:265-9.

6. Subissi L, Posthuma CC, Collet A, Zevenhoven-Dobbe JC, Gorbalenya AE, Decroly E, et al. One severe acute respiratory syndrome coronavirus protein complex integrates processive RNA polymerase and exonuclease activities. Proc Natl Acad Sci USA. 2014; 111: E3900-9.

7. Wang C, Horby PW, Hayden FG, Gao GF. A novel coronavirus outbreak of global health concern. Lancet. 2020; 395: 470-3.

8. Korber B, Fischer WM, Gnanakaran S, et al. Tracking changes in SARS-CoV-2 spike: evidence that D614G increases infectivity of the COVID-19 Virus. Cell 2020; 182(4): 812.e19-827.e19.

9. European Centre for Disease Prevention and Control. Detection of new SARS-CoV-2 variants related to mink - 12 November 2020. ECDC: Stockholm; 2020.

10. Public Health England. Investigation of novel SARSCoV-2 variant, Variant of Concern 202012/01 Technical briefing 2- 28 December 2020. PHE: London; 2020

11. World Health Organization. https://www.who.int/csr/ don/31-december-2020-sars-cov2-variants/en/

12. Cao Y, Li L, Feng Z, Wan S, Huang P, Sun X, Wen F, Huang X, Ning G, Wang W. Comparative genetic analysis of the 
novel coronavirus (2019-nCoV/SARS-CoV-2) receptor ACE2 in different populations. Cell Discov. 2020; 24: 6:11.

13. Wu Y, Feng Z, Li P, Yu Q. Relationship between ABO blood group distribution and clinical characteristics in patients with COVID-19. Clin Chim Acta. 2020;509:220-3.

14. Hou Y, Zhao J, Martin W, et al. New insights into genetic susceptibility of COVID-19: an ACE2 and TMPRSS2 polymorphism analysis. BMC Med. 2020;18(1):216

15. Kuo CL, Pilling LC, Atkins JL, Et al. APOE e4 genotype predicts severe COVID-19 in the UK Biobank community cohort. J Gerontol A Biol Sci Med Sci. 2020; glaa131.

16. Nguyen A, David JK, Maden SK, et al. Human leukocyte antigen susceptibility map for severe acute respiratory syndrome coronavirus 2. J Virol. 2020;94(13):e00510-20

17. Hou Y, Zhao J, Martin W, et al. New insights into genetic susceptibility of COVID-19: an ACE2 and TMPRSS2 polymorphism analysis. BMC Med. 2020;18(1):216.

18. Ellinghaus D, Degenhardt F, Bujanda L, et al. Genomewide association study of severe COVID-19 with respiratory failure. N Engl J Med. 2020;NEJMoa2020283 